\title{
DIEZ AÑOS DE EXCELENCIA PUBLICITARIA. LA RADIO EN EL FESTIVAL DE SAN SEBASTIÁN-EL SOL (1996-2006)
}

\section{$M^{\underline{a}}$ Teresa Piñeiro ${ }^{1}$}

\section{RESUMEN}

Los festivales constituyen un escaparate de la producción de una agencia publicitaria. El análisis del palmarés de los certámenes más prestigiosos del sector es suficiente para comprobar la relevancia de agencias, medios y marcas. En efecto, aunque la radio forma parte de los medios publicitarios clásicos, su presencia y participación en los festivales supone un reflejo de su escasa relevancia en el contexto actual.

El objeto de este trabajo ha sido el de conocer la presencia y evolución de la publicidad radiofónica en EI Sol. Un análisis que se ha efectuado teniendo en cuenta tanto el número de categorías presentes en su palmarés, como el volumen de piezas presentadas.

PALABRAS CLAVE: Publicidad - Radio-Festivales - Publicidad radiofónica - El Sol Excelencia publicitaria

\section{TEN YEARS OF ADVERTISEMENT EXCELLENCE. THE RADIO IN SAN SEBASTIAN-EL SOL FESTIVAL (1996-2006).}

\section{ABSTRACT}

Festivals are showcases for the production of advertising agencies. The analysis of the prize list of the most prestigious competitions in the sector is good for realizing the relevance of agencies, media and brands. Although radio is part of the classical advertising media, its presence and participation in festivals reflects the lack of relevance in the present context. The aim of this work is to approach the presence and evolution of radio advertising in El Sol festival. This analysis was performed taking into account both the number of categories of the prize list, and the amount of pieces submitted.

KEY WORDS: Advertising - Radio-Festivals - Radio advertisement - El Sol Advertisement excellence

\section{INTRODUCCIÓN}

\footnotetext{
${ }^{1} M^{\mathbf{a}}$ Teresa Piñeiro: Universidad de Vigo/ Pontevedra/ España. M.terepo@gmail.com
} 
Los certámenes publicitarios constituyen un escaparate de la industria de un determinado país y de la producción de las agencias publicitarias. Estos festivales constituyen un marco excepcional para dar a conocer aquellos anuncios, acciones o campañas de mayor calidad. Se tratan de acontecimientos únicos que permiten a los publicitarios "la promoción de sus mejores y más originales producciones mediante su participación en esa auténtica exposición internacional” (Ferrer Roselló, 1999: 119). Las piezas premiadas y las que se que presentaron su candidatura en los festivales publicitarios constituyen una muestra relevante de la actividad del sector en un año concreto.

Asimismo, como subrayan Till y Baack (2005), estos premios permiten reconocer las tendencias de la publicidad, tanto desde la perspectiva de la creatividad como de los medios-soportes empleados. La presencia del medio radiofónico en el palmarés de los certámenes de mayor prestigio podría constituir un indicador de la consideración de la publicidad sonora en un momento concreto. El presente trabajo ha tenido por objeto conocer la presencia y participación de la publicidad radiofónica en el Festival Publicitario de San Sebastián y -posteriormente- en El Sol en el período comprendido entre 1996 y 2006.

Se parte de la hipótesis de que la presencia de la radio y su participación dentro del palmarés del festival permite señalar la consideración del medio radiofónico en el contexto publicitario español y su evolución. Una hipótesis que sitúa al presente estudio en la línea de los trabajos académicos en torno a los festivales publicitarios del sector. Algunos de dichos trabajos son el de Helgesen (1994) sobre las piezas premiadas y su relación con la agencia, el de Angy y Low (2000) que relaciona creatividad y eficacia, o el de Beltramini y Blasko (1986) que profundiza en las características de los anuncios premiados. Otros estudios han cambiado este enfoque, de las piezas premiadas a los jurados (White y Smith, 2001) o -incluso- al público (Till y Baack, 2005).

\section{OBJETIVOS}

Los objetivos de este trabajo se desprenden de la introducción precedente. Si los festivales publicitarios constituyen el punto de encuentro de la excelencia publicitario del momento, la presencia de las radio podría suponer una mayor o menor consideración del medio para agencias y anunciantes. En este sentido, se estableció como principal objetivo el conocer la participación de la publicidad radiofónica a lo largo de una década con presencia en el Festival Publicitario de San Sebastián. Festival que, a partir de 2003, se amplió a la publicidad latina pasando a denominarse El Sol, Festival Iberoamericano de la Comunicación Publicitaria.

Para la consecución de este objetivo se establecieron una serie de objetivos específicos:

Conocer la presencia de la radio en el palmarés de este festival, número y tipo de categorías premiables, así como su año de introducción.

Analizar evolución de la participación de piezas radiofónicas en El Sol. Desde la producción nacional de e Festival Publicitario de San Sebastián, a la inclusión de la publicidad iberoamericana en EISol. 
Determinar el número de premios entregados entre las piezas radiofónicas presentadas a El Sol.

\section{METODOLOGÍA}

Para desarrollar el presente trabajo se ha tomado como muestra de estudio el Festival Publicitario de San Sebastián en el período comprendido entre 1996, año de creación del galardón de radio, y 2006. Esta selección implicó el registro de una década completa de presencia de la publicidad radiofónica en el certamen. Una década en la que el festival pasó de ser un certamen nacional (1996-2002) a abrirse a la publicidad del ámbito latino (2003-2006).

Partiendo del análisis de contenido como método de investigación, se efectuó un análisis cuantitativo de la presencia de la radio en el Festival basándose en las siguientes variables: participación, evolución dentro del palmarés -año de creación, ampliación o modificación categorial-y premios entregados.

\section{FESTIVALES PUBLICITARIOS}

Los festivales publicitarios se han convertido en el mejor escaparate de la excelencia publicitaria. Cada año las mejores campañas y piezas publicitarias se pasean por la alfombra roja de Cannes, San Sebastián o cualquier otro certamen publicitario de referencia.

Según señalan Till y Baack (2005) existen más de 500 eventos publicitarios cada año; unos certámenes que van desde pequeños premios sectoriales -por ejemplo los premios Aspid, de publicidad farmacéutica- a verdaderos espectáculos internacionales como el Festival de Cannes o el decano Echo Awards (creado en 1929).

La participación en estos certámenes permite a creativos y agencias salir del anonimato. Como señala De León (1999: pág. 3) al contrario que sucede con otros artistas, los publicitarios carecen del reconocimiento social.

(...) mientras sus obras, los anuncios, se difunden masivamente, e incluso algunas frases de los mismos hacen fortuna entre la gente, engrosando el gracejo popular, ellos apenas salen a escena, los mejores de entre ellos no son conocidos más allá del propio sector publicitario; serán sólo los festivales publicitarios (...) los que permitan escenificar su triunfo.

La publicidad de calidad vende, y no sólo desde una perspectiva comercial.

La comunicación publicitaria cuenta con gran atractivo para el público, llegando a constituir una fórmula más de entretenimiento. Esta consideración de los mensajes publicitarios ha supuesto un traspaso en la concepción de su público, que pasa de ser el destinatario de productos y servicios al consumidor de productos culturales (Caro, 1995). 
En este contexto los festivales constituyen una fórmula única de hacer publicidad de la publicidad; un catálogo en el que el sector ofrece una selección de piezas de calidad "desde su punto de vista" (Rubio de Miguel, 1997).

La mejor tarjeta de presentación para un publicitario o agencia es la de contar con algún galardón de un certamen de referencia. Esta circunstancia genera en los autores una necesidad imperante por hacerse con algunos de los premios; necesidad que Álvarez-Debans (2004) denominó "festival dependencia".

En cualquier caso, "Un galardón es [o debería ser] el reconocimiento a la labor bien hecha, a un buen y creativo trabajo; no es la finalidad del mismo". (Muela, 2001: pág. 254).

\subsection{La publicidad radiofónica a concurso}

Desde sus inicios la radio se configuró como un medio publicitario de gran potencial. En el momento actual se sitúa el segundo medio con mayor audiencia (AIMC, 2008) y el cuarto en inversión publicitaria $(678,1 \mathrm{M} €)$.

Sin embargo la radio está inmersa en una crisis publicitaria, propiciada por la infrautilización del medio y la escasa innovación en sus formatos. Un estancamiento que, como señala Rodero (2008) le ha pasado factura.

La radio ha sido considerada como un medio publicitario de segundo orden (Barbeito y Fajula, 2005). "Las agencias de publicidad siempre le vendieron a los anunciantes sus bobinas de televisión, en las que no había nada de radio ni prensa, sino sólo una sucesión de anuncios de televisión, la mayoría premiados, que componen ese background estelar que vende a coste de León de Oro" (Control: 1998).

Un ejemplo de la infravaloración del medio publicitario se puede observar en la tardía incorporación de la categoría de radio al Festival Publicitario de Cannes, coincidiendo con la quincuagésima segunda edición del certamen.

Sin embargo, en la última década hay autores como Betés (2002) o Merayo (2003) que destacan el incremento en la calidad de la producción publicitaria radiofónica. Un incremento que ha llevado su reconocimiento en los principales certámenes de referencia del sector.

\section{EL FESTIVAL PUBLICITARIO DE SAN SEBASTIÁN}

El festival de San Sebastián, actualmente conocido como "El Sol", inició su andanza en 1984. En este año la Asociación Española de Agencias de Publicidad (AEAP) planteó la necesidad de crear un festival para impulsar el producto creativo publicitario español (Rubio, 2003).

No sería hasta 1986 cuando, de la mano de Xavier Oliver, tuvo lugar la primera edición del llamado Festival Publicitario de Cine y Televisión en Marbella "de ahí (...) la denominación de los premios Sol” (Rubio, 2003). 
La siguiente edición de este festival se celebró en San Sebastián, un emplazamiento que se ha mantenido hasta la actualidad. En 1993 este certamen incorporó la publicidad gráfica, pasando a denominarse Festival Publicitario de San Sebastián, título con el que se convirtió en un referente de la creatividad publicitaria española.

Sin embargo, habría que esperar hasta la undécima edición del certamen para que el festival contase con participación de la publicidad radiofónica.

\subsection{Participación y evolución de la publicidad radiofónica en el certamen}

En 1996 el ya célebre certamen incorporó por vez primera una categoría específica de publicidad radiofónica, a la que se sumó el gran premio del festival. Estas categorías se mantuvieron invariables hasta 2004, año en el que se crearon dos galardones nuevos: mejor uso del medio y el premio COPE al mejor uso creativo.

La publicidad para el medio radiofónico no tuvo una evolución semejante a los otros dos soportes tradicionales: el audiovisual y el gráfico. Esta diferencia de participación en la categoría radiofónica, frente al incremento exponencial y continuado en las restantes categorías de publicidad, se mantuvo durante casi todo el período de estudio.

En efecto, pese a la existencia de fluctuaciones entre ediciones, la participación en la categoría de radio del festival de San Sebastián se ha situado en torno a las 131 piezas/año, teniendo su valor máximo en el año 2000 (168 piezas) y el mínimo en 2002 (91 cuñas radiofónicas).

Gráfico 1. Evolución en el número de piezas publicitarias presentadas al certamen (publicidad total y radiofónica) entre 1996-2006.

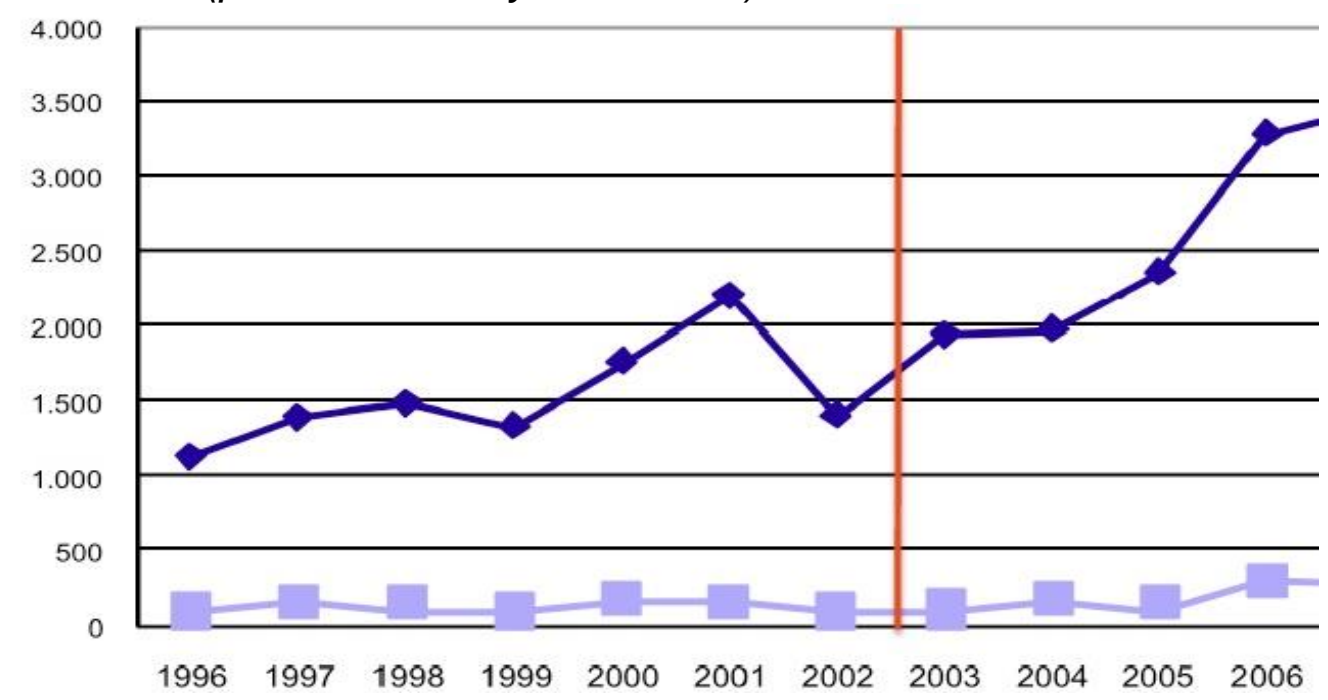

Fuente: Elaboración propia.

Esta tendencia, que se extendió a las primeras ediciones de El Sol, parece cambiar en el año 2006, cuando el número de piezas radiofónicas presentadas al certamen superaron -por vez primera- las 300 cuñas; el doble de la participación de años 
anteriores.

Gráfico 2. Evolución número de piezas radiofónicas presentadas al Festival entre 1996-2006.

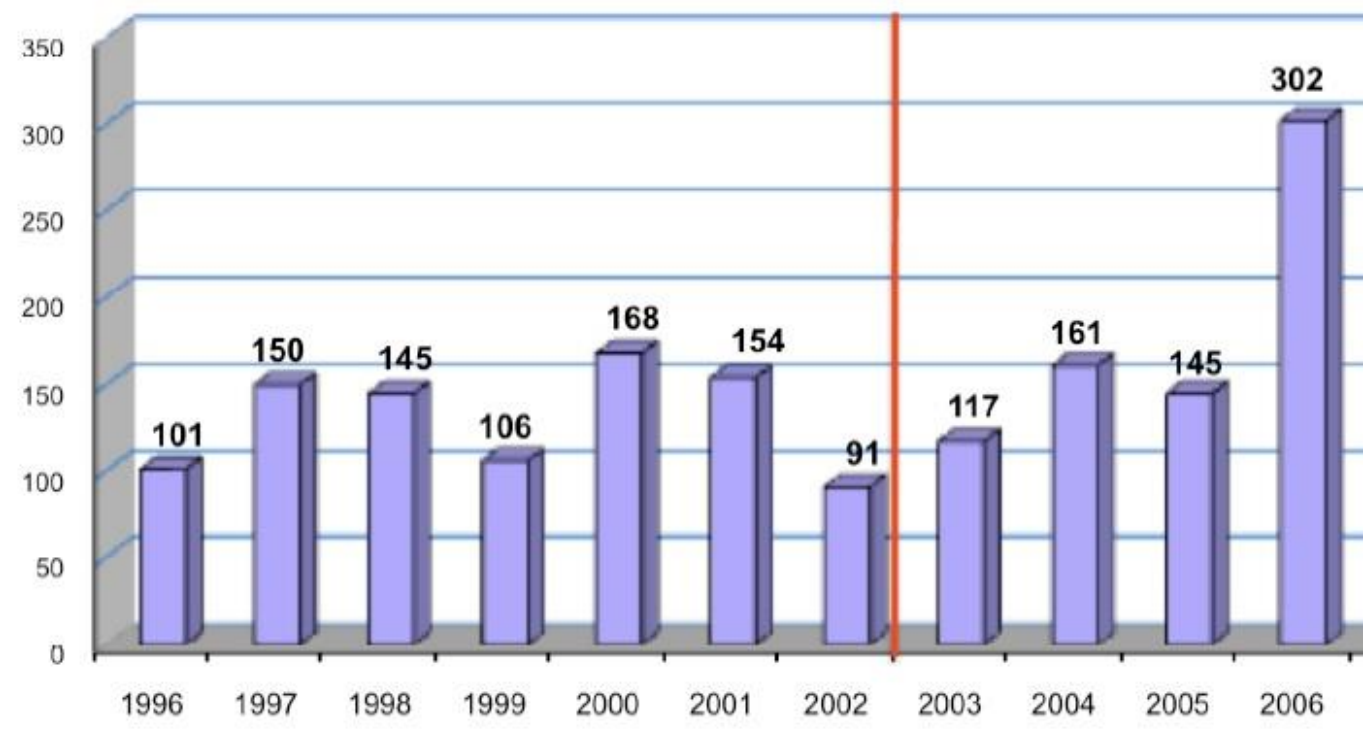

Fuente: Elaboración propia

La limitada participación de la publicidad radiofónica relegó al medio sonoro a una única categoría, en lo que se refiere a los productos anunciados, hasta la vigésima segunda edición (2007).

Como señalaba Rubio (2003: pág. 100)

Un anuncio de coches no compite con un anuncio de electrodomésticos, sino que existe una serie de categorías de productos. Entonces, tanto en cine, en televisión y en gráfica tenemos 14 categorías, las mismas, que van desde bebidas alcohólicas, artículos de fumador, electrónica de consumo, alimentación, medios de comunicación, automóviles... Tratamos en esas 14 categorías de recoger todos los productos que se anuncian en el mercado. En radio tenemos una única categoría, por cuanto el número de cuñas que actualmente se estrena en el festival aún no nos permite agruparlas por categorías.

En la decimoséptima edición, el Festival Publicitario de San Sebastián se abrió a Portugal, América Latina y los Estados Unidos hispanos. De este modo "El Sol" pasó a ser la cita por excelencia de la publicidad de habla hispana y San Sebastián se postuló como la capital de la creatividad Iberoamericana, con la consecuente promoción internacional que lo situó al nivel de festivales de mayor antigüedad.

Si bien la apertura del certamen a los países de habla hispana no propició el incremento inmediato en el número de piezas presentadas, sí supuso una de las causas del salto cualitativo acontecido en su vigésima primera edición (2006), que llevó a la ampliación de categorías y premios -para el medio radiofónico- en el 
certamen de 2007.

Esta proyección internacional se logró manteniendo una máxima de calidad tanto en la elección de los jurados como en los premios. "En este Festival tomamos la decisión de que el jurado debería estar integrado por profesionales del área creativa. Queremos además que sean creativos de alto nivel y personas indiscutidas, lo cual es difícil, sobre todo en la firma del Presidente" (Rubio, 2003: pág. 100).

\subsection{Evolución de los premiados}

El premio a la calidad y a la excelencia publicitaria ha llevado, en diversas ocasiones, a declarar desiertos algunos de los premios y categorías del festival. En este sentido, Juan Capmany -Presidente de DDB España- ante el elevado número de galardones desiertos en la XII del Festival de San Sebastián destacaba que la creatividad si no sirve para vender más, o para mejorar la simpatía hacia una determinada marca, no sirve para nada (ABC, 1999).

En el caso de la radio, si se toma como referencia el número total de premios posibles, y el de galardones entregados, se puede observar una cierta concordancia entre ambas variables, especialmente si se compara con la publicidad televisiva presentada a este certamen.

En efecto, en la mayoría de las ediciones objeto de estudio, las piezas publicitarias radiofónicas se hicieron con el total de premios a los que candidateaban (43\%) o con más del ochenta por ciento de los mismos (22\%).

Tabla 1. Número de categorías de radio, galardones y premios entregados (19962006).

\begin{tabular}{|c|c|c|c|c|}
\hline & CATEGORÍAS RADIO & $\mathrm{N}^{0}$ TOTAL GALARDONES & PREMIOS ENTREGADOS & $\%$ PREMIOS RADIO \\
\hline 1996 & 2 & 4 & 4 & $100 \%$ \\
\hline 1997 & 2 & 4 & 4 & $100 \%$ \\
\hline 1998 & 2 & 4 & 4 & $100 \%$ \\
\hline 1999 & 2 & 4 & 4 & $100 \%$ \\
\hline 2000 & 2 & 4 & 3 & $75 \%$ \\
\hline 2001 & 2 & 4 & 3 & $75 \%$ \\
\hline 2002 & 2 & 4 & 4 & $100 \%$ \\
\hline 2003 & 2 & 4 & 2 & $50 \%$ \\
\hline 2004 & 4 & 6 & 5 & $83 \%$ \\
\hline 2005 & 4 & 6 & 6 & 100 \\
\hline 2006 & 3 & 5 & 4 & 80 \\
\hline
\end{tabular}

Fuente: Elaboración propia

Son diversos los criterios para otorgar -o no- un determinado premio. Se puede marcar la excelencia publicitaria, y en función de este máximo valorar los trabajos presentados, u otorgar todos los premios escogiendo las mejores piezas entre el total White y Smith (2001) señalan que, si los jurados determinan que la publicidad 
es acreedora de este galardón, se puede considerar al jurado como una medida apropiada de la creatividad.

Desde la perspectiva del medio radiofónico resulta complejo valorar, en una única categoría, la creatividad publicitaria de productos y servicios tan diferentes como bancos y bebidas alcohólicas.

En esta línea, el elevado porcentaje de premios entregados podría considerarse más una consecuencia del limitado número de galardones dedicados al medio radiofónico que de la creatividad. No obstante, cada vez que un importante número de categorías queda desierto -como sucedió con la radio en 2007- son numerosas las voces que subrayan la existencia de una crisis de creatividad publicitaria.

\section{CONCLUSIÓN}

El análisis efectuado ha permitido determinar la infrarrepresentación de la publicidad radiofónica en el certamen celebrado en San Sebastián respecto otros soportes publicitarios clásicos como el televisivo o el gráfico.

Esta infrarrepresentación va a reflejarse en dos aspectos diferentes, aunque estrechamente relacionados: limitada inscripción de piezas radiofónicas al certamen y escasa presencia de la radio en el palmarés del festival, en el que la publicidad radiofónica se valora en una única categoría.

A pesar de su gran potencial como medio publicitario, dada su elevada penetración en el contexto español, su escasa presencia en los festivales va a constituir un indicador de la limitada consideración que la industria publicitaria nacional tiene de la radio. Sin embargo, la apertura del festival a otras industrias publicitarias ha propiciado una mayor proyección de la publicidad radiofónica tanto en lo que respecta a su participación como al número de galardones

\section{REFERENCIAS:}

\section{Libros:}

Ferrer Roselló, C. (1999). La publicidad, motor creativo mundial: festivales de Cannes y San Sebastián, en Fernández-Areal, M. (Dir). La publicidad en televisión (pp. 113-122). Pontevedra: Deputación Provincial de Pontevedra.

Merayo, A. (2003). Para entender la radio. Salamanca: Publicaciones Universidad Pontificia de Salamanca.

Muela, C. (2001). La publicidad radiofónica en España.Madrid: Ediciones Universitarias.

Rubio de Miguel, C. (2003). La publicidad local y elFestival del Sol, En R. López Lita, F. Fernández Beltrány A. Durán. La publicidad local (pp. 97-104). Castellón de 
la Plana: Universitat Jaume I.

\section{Artículos en revistas:}

Álvarez-Debans, N. (2004). Vedettismo de los Creativos en la Comunicación Publicitaria, en Razón y Palabra, vol. 39. Consultado el 6 de enero de 2008. Disponible en línea en: http://www.razonypalabra.org.mx/anteriores/n39/nalvarez.html

Ang, S.H. y Low, S. (2000). Exploring the dimensions of ad creativity, en Psychology and Marketing, vol. 17, no 10, p. 835-854.

Barbeito, M. L. y Fajula, A. (2005). La radio publicitaria: el peso del inmovilismo, en Quaderns del CAC, vol. 22, p. 49-62.

Beltramini, R. E. y Blasko, V, J. (1986). An Analysis of Award-Winning Advertising Headlines, en Journal of Advertising Research, vol. 26, nำ 2, p. 48-52.

Caro, A. (1995). Cinco años que cambiaron la publicidad, en Anuncios, vol. 46, p. 32-38.

Control (1998). La radio la gran desconocida, en Control, vol. 425, p. 23.

Gámez, M. J., González Moreno, L. G. y Rivas, A.M.(2004). Imaginarios culturales en la publicidad internacional: Cannes 1998-2002. Cultura, lenguaje y representación, en Revista de estudios culturales de la Universitat Jaume, vol. I, no 1, p. 29-42.

Helgesen, T. (1994). Advertising awards and advertising agency performance criteria, en Journal of Advertising Research, vol. 4, n 34, p. 43-59.

De León, J. L. (1999). Creativos publicitarios: una visión del mundo, en Zer, nº 7, p. 161-179.

Rodero, E. (2008). Publicidad en radio: Publicidad, sí, pero no radiofónica, en Área abierta, no 20, p. 1-16.

Till, B. y Baack, D. W. (2005). Does creative advertisingmatter? Journal of Advertising, vol. 3, no 34, p. 47-57.

White, A. y Smith, B. (2001). Assessing AdvertisingCreativity Using the Creative Product Semantic Scale, en Journal of Advertising Research, vol. 41, № 6, p. 27-34.

\section{Recursos electrónicos:}


AIMC (2008). Resumen general de resultados EGM. Febrero a Noviembre de 2007.Consultado el 18 de enero de 2008. Disponible en línea en: http://www.aimc.es/aimc.php

INFOADEX (2008). Estudio Infoadex de la inversión publicitariaen España. Consultado el 18 de enero de 2008. Disponible en línea en: http://www.infoadex.es/infoadex.asp

La publicidad española en tela de juicio. (1999, junio 2).ABC. Consultado el 18 de enero de 2008. Disponible en línea en: www.abc.es. 